\title{
Lymphoid Interstitial Pneumonia as a Pulmonary Lesion of Idiopathic Plasmacytic Lymphadenopathy with Hyperimmunoglobulinemia
}

\author{
Keizo ToriI, Kenji Ogawa*, Yoshinori Kawabata**, Toyoharu YoKoI***, \\ Kenzo TAKagi and Taro MiWA*
}

\begin{abstract}
A case of idiopathic plasmacytic lymphadenopathy with hyperimmunoglobulinemia is reported. A 71-year-old man was admitted to the hospital because of an abnormal shadow on chest roentgenogram. Chest $\mathrm{X}$-ray taken on admission showed remarkable diffuse infiltration and pleural thickening. Laboratory examinations revealed an elevated total protein level of $10.1 \mathrm{~g} / \mathrm{dl}$, and a gammaglobulin level of $7.0 \mathrm{~g} / \mathrm{dl}$ including $6,790 \mathrm{mg} / \mathrm{dl} \mathrm{IgG}$. Mediastinal lymphadenopathy was observed on a chest CT. The patient underwent open lung biopsy. Heavy infiltration of lymphocytes and plasma cells were seen in the moderately fibrotic pulmonary interstitium. LIP was diagnosed. Lymph node biopsy was also performed. Follicular hyperplasia with prominent germinal centers and plasma cell proliferation in the interfollicular area were seen. Treatment with prednisolone resulted in an improvement in the chest $\mathrm{X}$-ray findings, as well as a diminished polyclonal hypergammaglobulin level.
\end{abstract}

(Internal Medicine 33: 237-241, 1994)

Key words: LIP, IPL, Castleman's disease, open lung biopsy

\section{Introduction}

Carrington and Liebow reported 5 cases of an entity that they called lymphocytic interstitial pneumonia (1). After their report, some of these cases were found to be associated with other immunologic disorders, such as Sjögren's syndrome (2), myasthenia gravis (3) and pseudolymphoma (4). It has also been reported that this disease is frequently associated with dysproteinemia, monoclonal or polyclonal hyperimmunoglobulinemia and hypoimmunoglobulinemia $(3,5-8)$. Liebow and Carrington have reviewed additional cases of the disease, which they later termed lymphoid interstitial pneumonia (LIP) (9). In Japan, a clinicopathological entity, idiopathic plasmacytic lymphadenopathy with hyperimmunoglobulinemia (IPL), in which a remarkable number of plasma cells infiltrate into systemic lymph nodes as well as lungs and skin, has been proposed (10). Although this disease is very similar to multicentric Castleman's disease, whether or not these two diseases are identical still remains uncertain. However, associa- tion of pulmonary involvement in patients with Castleman's disease has not been reported. Here we describe a case of lymphoid interstitial pneumonia (LIP) associated with IPL. We confirmed LIP by open lung biopsy, followed by an immunohistochemical method. We have treated the patient with a moderate dose of prednisolone and observed apparent improvement on chest roentgenogram, and hypergammaglobulinemia and pulmonary function. This is the second report of LIP associated with IPL, confirmed by open lung biopsy; the first report is that of Akanuma's (11).

\section{Case Report}

The patient is a 71-year-old Japanese male who was admitted to Higashi Nagoya National Hospital on April 22, 1991 following a recent medical examination. Diffuse interstitial infiltration on both upper lung fields and pleural thickening were first pointed out on his X-ray findings in 1979. He was treated with isoniazid and ethambutol for 2 months, then

From the Second Department of Internal Medicine, School of Medicine, University of Nagoya, Nagoya, *the Division of Respiratory Medicine, Department of Internal Medicine, Higashi Nagoya National Hospital, Nagoya, **the Division of Pathology, Research Institute of Tuberculosis, Japan Anti-tuberculosis Association, Kiyose, Tokyo, and ***the Department of Clinical Laboratory, Nagoya National Hospital, Nagoya

Received for publication August 27, 1993; Accepted for publication February 17, 1994

Reprint requests should be addressed to Dr. Kenji Ogawa, the Division of Respiratory Medicine, Department of Internal Medicine, Higashi Nagoya National Hospital, 5-101 Umemorizaka, Meitoh-ku, Nagoya, 465 
isoniazid alone for 4 months following the 1979 checkup. His chest roentgenogram had not shown any remarkable changes for 5 years. No further follow-up was performed until 1989 at the time of his next medical checkup. On his chest X-ray, infiltration and pleural thickening appeared to have worsened compared with the 1979 film. Further examinations, however, were not performed at that time. Recently he felt slight exertional dyspnea. The chest roentgenogram taken on admission (Fig. 1) showed diffuse infiltration on both upper lung fields, pleural thickening and the loss of the lung volume compared with the 1979 film. Dry rales were slightly heard on the lower back. Clubbing of the fingers and cyanosis were absent. Liver and spleen were not palpable, and no skin rash nor superficial lymphadenopathy was observed. Laboratory findings on admission are presented in Table 1. Pulmonary function study revealed mild restrictive impairment, and arterial blood gas analysis showed mild hypoxemia. The erythrocyte count was 383 million and the sedimentation rate was $130 \mathrm{~mm} / \mathrm{h}$. Total protein was $10.1 \mathrm{~g} / \mathrm{dl}$, albumin $3.1 \mathrm{~g} / \mathrm{dl}$ and globulin $7.0 \mathrm{~g} / \mathrm{dl}$. The electrophoretic pattern showed: albumin $35.7 \%, \alpha_{1}$ globulin $3.1 \%, \alpha_{2}$ globulin $7.2 \%$, $\beta$ globulin $6.5 \%$, and $\gamma$ globulin $47.5 \%$. The immunoglobulin fraction showed a marked increase in IgG. Immunoelectrophoresis showed polyclonal gammopathy. Cutaneous reaction to PPD was positive

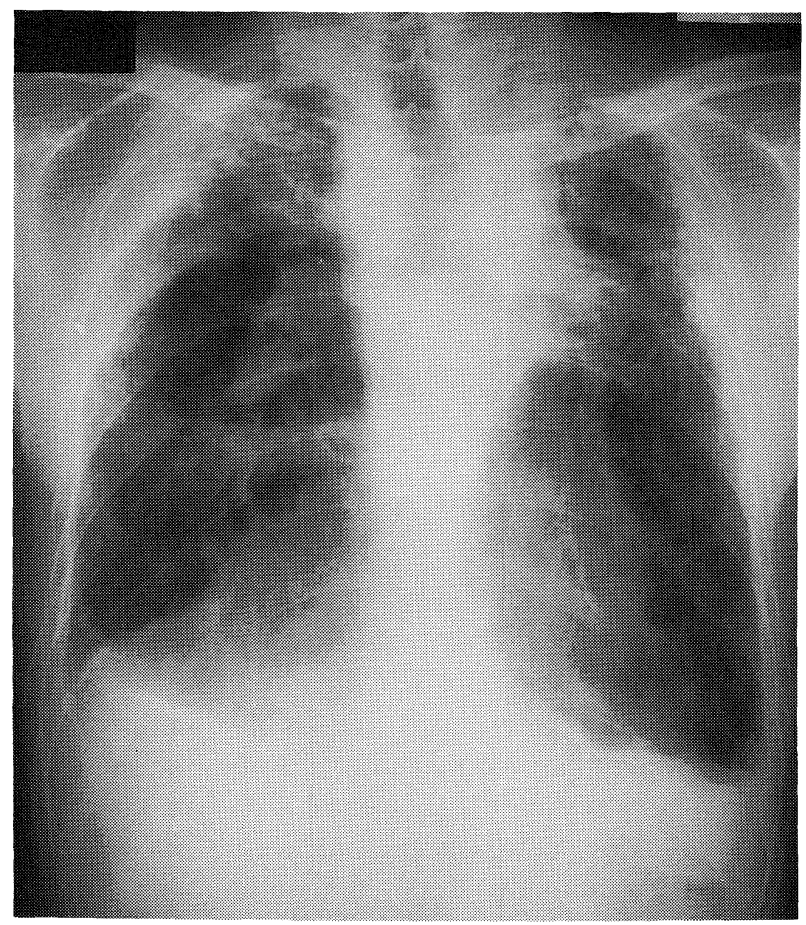

Fig. 1. Chest roentgenogram taken at admission. Marked infiltration, together with pleural thickening was seen on both lung fields.

Table 1. Laboratory Data

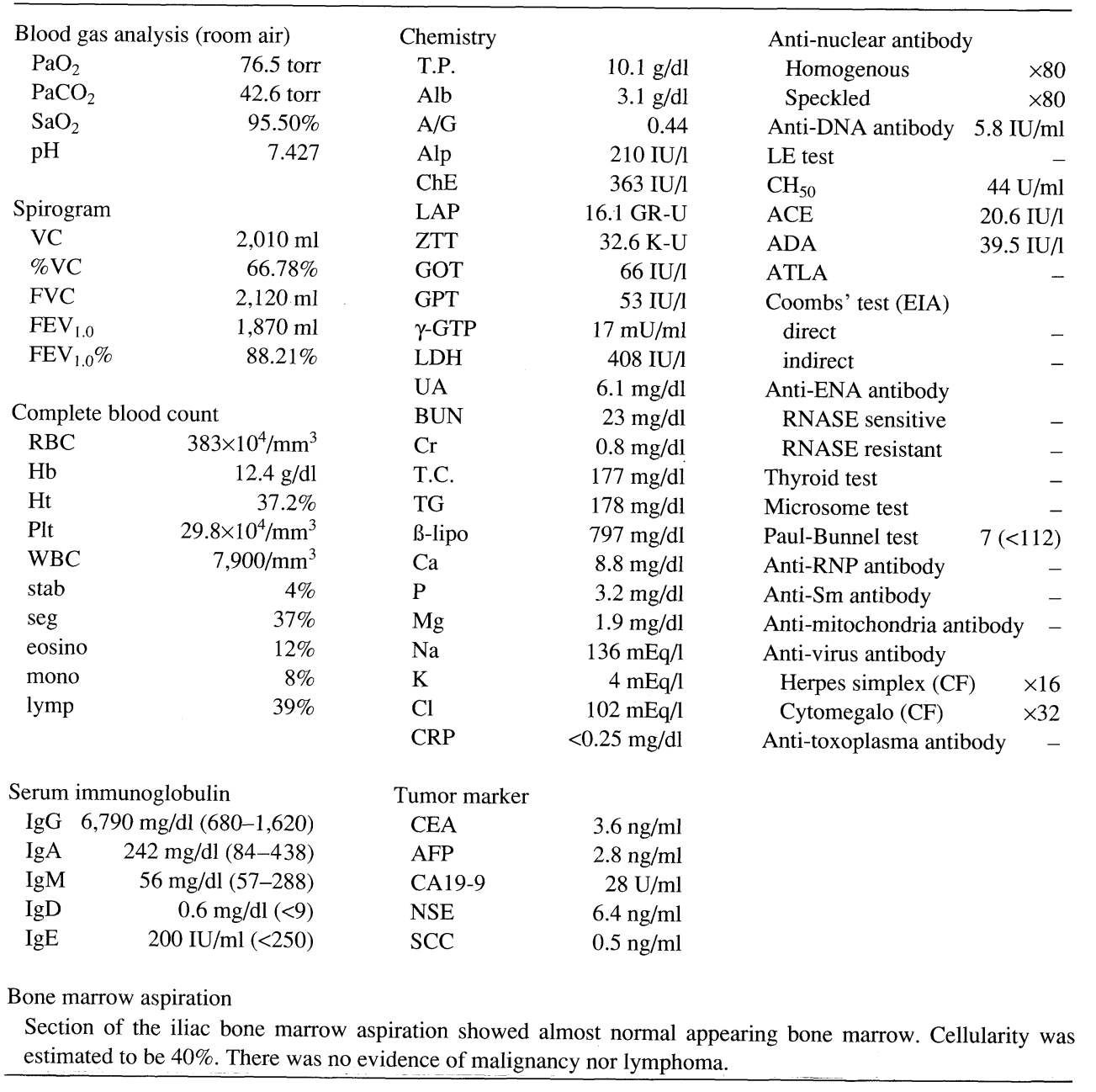


$(15 \times 15 \mathrm{~mm})$. Definite evidence suggesting viral infection or autoimmune disease was not found except for slightly elevated titers of anti-viral antibodies and anti-nuclear antibodies. On repeated urinalyses, no proteinuria could be detected and Bence Jones protein was negative. On chest computed tomography, mediastinal lymphadenopathy was observed. Transbronchial lung biopsy was performed 7 days after admission, which showed infiltration of inflammatory cells to alveolar septum and mild fibrotic changes. Bronchoalveolar lavage (BAL) was also performed. Analysis of BAL fluid cells on centrifuge preparations revealed an increased proportion of lymphocytes (17\%). Thirty-four days after admission, open lung biopsy was performed. The upper portion of the left thoracic cavity exhibited fibrous adhesion, so $\mathrm{S}^{8}$ was chosen as the biopsy site. The biopsy specimen from left $S^{8}$ showed patchy honeycombing, smooth muscle proliferation and diffuse infiltration of plasma cells and lymphocytes in the alveolar wall (Fig. $2 a$ and $2 b$ ). Similar lymphoplasmacytic infiltration and fibrosis, together with local exudation of fibrin were presented in the visceral pleura (Fig. 3). The lung specimen was also examined
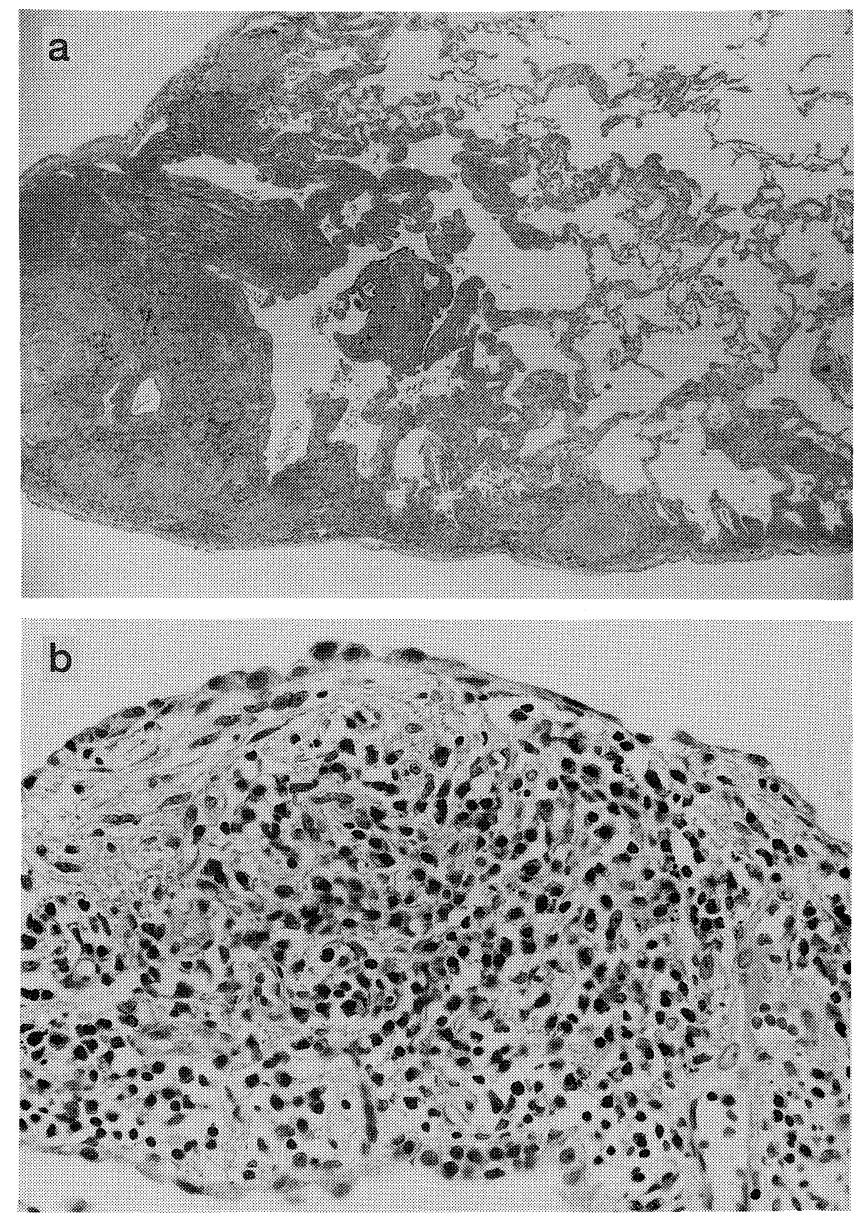

Fig. 2. a) Various degrees and stages inflammatory changes in the lung and pleura (HE stain, $\times 20$ ). b) Heavy infiltration of lymphocytes and plasma cells in the mildly fibrotic pulmonary interstitium (HE stain, $\times 400$ ). immunohistochemically using antisera against light chains of immunoglobulins ( $\kappa$ and $\lambda$ ), T lymphocytes and B lymphocytes (data not shown). Polyclonality of plasma cells and lymphocytes was observed. These findings were compatible to chronic fibrotic stage lymphoid interstitial pneumonia (LIP). Figure 4 shows a mediastinal lymph node taken at the open lung biopsy. Follicular hyperplasia with prominent germinal center and plasma cell proliferation in the interfollicular area were seen. To confirm implication of interleukin-6, IL-6 was measured using enzyme-linked immunosorbent assay (ELISA). Serum IL-6 level was, however, under the detection level. After establishment of the diagnosis, prednisolone was administered at an initial dosage of $40 \mathrm{mg} / \mathrm{day}$, which was reduced gradually over a 10 -week period. Chest roentgenogram taken on hospital day 154 revealed remarkable improvement of the pleural thickening and interstitial infiltration (Fig. 5). The serum total

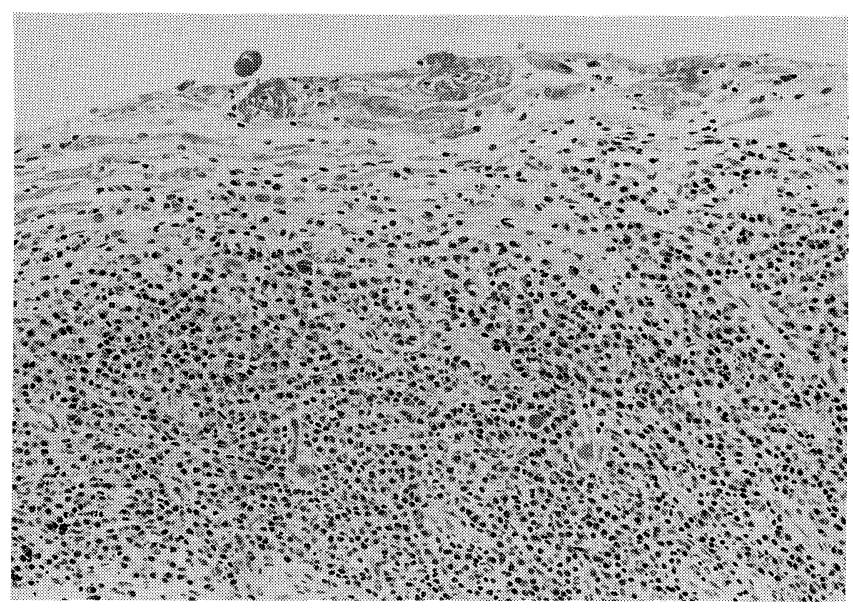

Fig. 3. Heavy diffuse infiltration of plasma cells and lymphocytes in the fibrotic pleura (HE stain, $\times 200$ ).

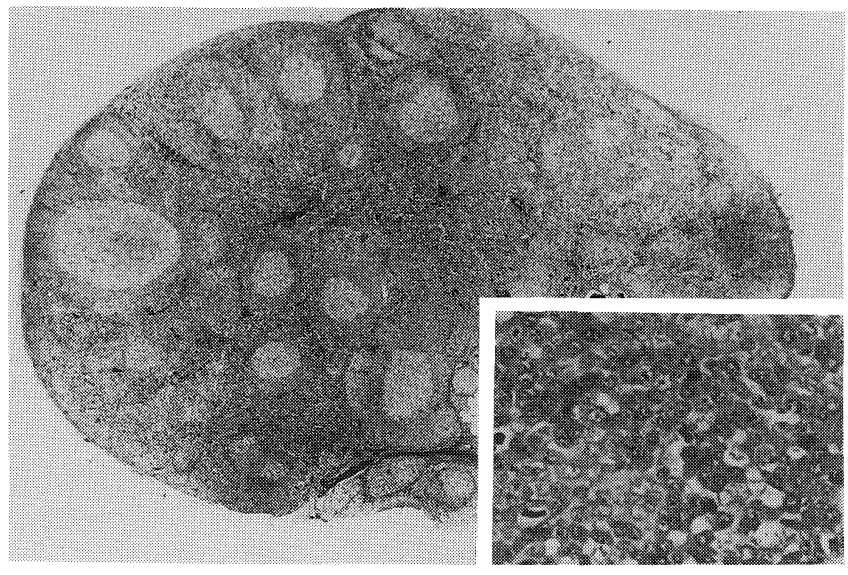

Fig. 4. Mediastinal lymph node. Follicular hyperplasia with prominent germinal center was seen (HE stain, $\times 4$ ). Plasma cells infiltration was observed in the interfollicular area (HE stain, $\times 400$ ). 


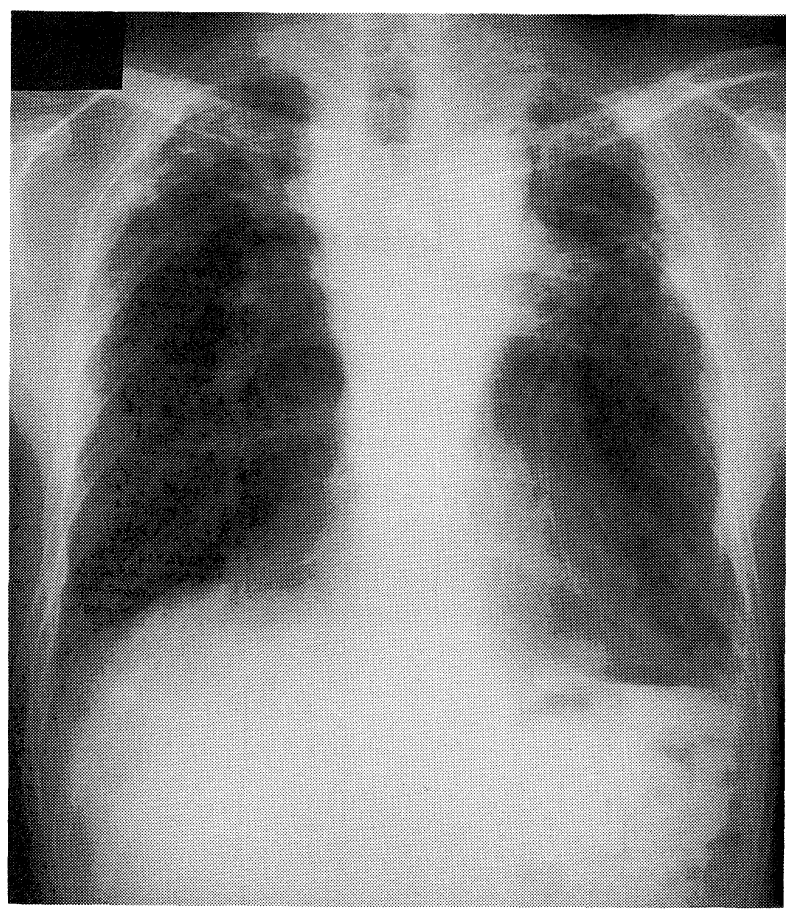

Fig. 5. Chest X-ray film taken after treatment. Improvement of the lung infiltration could be seen.

Table 2. Pulmonary Function Test and Blood Gas Analysis*

\begin{tabular}{lcc}
\hline & $\begin{array}{c}\text { before treatment } \\
(1991,4,23)\end{array}$ & $\begin{array}{c}\text { after treatment } \\
(1992,10,14)\end{array}$ \\
\hline Blood gas analysis & & \\
$\mathrm{PaO}_{2}$ (torr) & 76.5 & 73.6 \\
$\mathrm{PaCO}_{2}$ (torr) & 42.6 & 40.0 \\
$\mathrm{SaO}_{2}(\%)$ & 95.5 & 95.1 \\
$\mathrm{pH}$ & 7.427 & 7.421 \\
& & \\
Spirogram & & \\
VC $\left(\mathrm{cm}^{3}\right)$ & 2,010 & 2,480 \\
$\% \mathrm{VC}(\%)$ & 66.78 & 83.22 \\
$\left.\mathrm{FVC}_{(\mathrm{cm}}{ }^{3}\right)$ & 2,120 & 2,500 \\
$\mathrm{FEV}_{1.0}\left(\mathrm{~cm}^{3}\right)$ & 1,870 & 2,310 \\
$\mathrm{FEV}_{1.0} \%$ & 88.21 & 92.40 \\
\hline
\end{tabular}

*Blood gas analyses were performed on room air.

protein level and $\mathrm{IgG}$ decreased to $6.4 \mathrm{~g} / \mathrm{dl}$ and $2,050 \mathrm{mg} / \mathrm{dl}$, respectively. He was discharged from the hospital when the prednisolone was tapered to $10 \mathrm{mg}$ a day; he has been followed at this hospital without any symptoms. Pulmonary function test performed after treatment also revealed improvement (Table 2).

\section{Discussion}

Originally, LIP was reported as one type of chronic intersti- tial pneumonia (12). But Colby and Carrington have reported that many cases previously regarded as LIP or pseudolymphoma, are actually malignant lymphomas (13). It is difficult to distinguish LIP from low grade malignant lymphoma. Therefore in order to obtain an accurate diagnosis, open lung biopsy specimens should be examined immunohistochemically. In this case, we performed open lung biopsy and confirmed the polyclonality of plasma cell and lymphocytes by immunohistochemistry. Association of dysproteinemia with LIP might indicate the existence of immunological disorders at the base of this disease. Upper lobe infiltration and pleural thickening were also improved by steroid therapy, so we thought these lesions were also LIP.

Recently, Mori and colleagues have proposed a new entity that they termed idiopathic plasmacytic lymphadenopathy with hyperimmunoglobulinemia, or, IPL (10). According to their criteria, the characteristic of IPL are as follows; 1) Existence of polyclonal hyperimmunoglobulinemia and serum IgG level higher than 4,500 mg/dl without M-peak. 2) Existence of systemic superficial lymphadenopathy. The largest lymph node should be more than the width of a thumb or $1.8 \mathrm{~cm}$ in diameter. Histologically, the lymph node is characterized by marked infiltration of plasma cells without destruction of architecture. 3) Absence of other diseases associated with hyperimmunoglobulinemia. They reviewed 10 cases of IPL. They found the similarity between IPL and plasma cell type of giant lymph node hyperplasia. The major difference between them they mentioned was the existence of systemic lymphadenopathy, which was observed in patients with IPL. Although there was no superficial lymphadenopathy in the present case, mediastinal lymphadenopathy was observed, the histopathologic findings of which were just the same as IPL. Moreover pulmonary involvement in the present case is similar to that of IPL. Therefore, we regard the present case as a variant type of IPL. These facts suggest that IPL might be a predisposing factor of LIP (14).

There have been no reports that describe association of LIP in patients with Castleman's disease. Castleman's disease was originally defined as a solitary intra-mediastinal lymphadenopathy and elevated serum immunoglobulin level. Removal of this lymph node results in the decrease of serum immunoglobulin. Castleman's disease is now regarded as a functioning tumor which produces interleukin-6 (IL-6) (15). IL-6, a cytokine released from $\mathrm{T}$ lymphocytes, activates immunoglobulin production by B lymphocytes. Therefore, IL6 might also play some roles in the genesis of IPL. The serum level of IL-6, however, was not elevated in the present case.

Ohkubo and colleagues also reported a case of IPL (16). In their report, they summarized nineteen cases including their own, and found plasma cells to infiltrate into organs other than lymph nodes, mainly bone marrow and lungs. Most patients showed little or slight exacerbation in their clinical course. In our case, the onset of this disease is not clear and twelve years have passed since 1979 when an abnormality was first pointed out on chest X-ray findings. These facts suggest that IPL is a generalized benign lymphoproliferative disorder. The progres- 


\section{LIP as a Pulmonary Lesions of IPL}

sion of symptoms after treatment is also very slow. As for the treatment of this disease, here prednisolone administration did reduce the serum total protein but interruption of the administration caused immunoglobulins to elevate. Because the incidence of LIP is rare, the present case appears to be of interest to many physicians.

Acknowledgment: The writers thank Prof. Thomas V. Colby of the Mayo Clinic, Department of Laboratory Medicine and Pathology, for his histological evaluation and review of the manuscript. They also thank Dr. Ken-ichi Iida for his technical assistance.

\section{References}

1) Carrington CB, Liebow AA. Lymphocytic interstitial pneumonia. Am J Pathol 1966; 36a (Abstract).

2) Strimlan CV, Rosenow EC, Divertie MB, Harrison EGJ. Pulmonary manifestations of Sjögren's syndrome. Chest 70: 354, 1976.

3) Montes M, Tomashi Jr BT, Noehren TH, Culver GJ. Lymphoid interstitial pneumonia with monoclonal gammopathy. Am Rev Respir Dis 98: 277, 1967.

4) Gibbs AR, Seal RME. Primary lymphoproliferative conditions of lung. Thorax 33: 140, 1978.

5) Rosce C, Young J, Tillman RL, Burton AF, Sampson CC. Lymphoid interstitial pneumonia with polyclonal gammopathy. J Natl Med Assoc 61: $310,1969$.

6) Levinson AI, Hopewell PC, Stites DP, Spitler LE, Fundenberg HH. Coexistent lymphoid interstitial pneumonia, pernicious anemia, and agammaglobulinemia. Arch Intern Med 136: 213, 1976.
7) Strimlan CV, Rosenow III ECR, Weiland LH. Lymphocytic interstitial pneumonitis. Ann Intern Med 88: 616, 1978.

8) Liebow AA, Carrington CB. Diffuse pulmonary lymphoreticular infiltrations associated with dysproteinemia. Med Clin NA 57: 809, 1973.

9) Liebow AA. New concepts and entities in pulmonary diseases. in: The Lung, Liebow AA, Smith DE, Eds. Williams \& Wilkins, Baltimore, 1968.

10) Mori S, Mohri N, Uchida T, Shimamine $T$. The cases of idiopathic plasmacytic lymphadenopathy with polyclonal hyperimmunoglobulinemia. Nippon Mounaikei Gakkai Kaishi 20: 85, 1981 (in Japanese).

11) Yasuo Akanuma, Riichiro Mikami, Masami Nakamura et al. A case of idiopathic plasmacytic interstitial pneumonia with hyperproteinemia. Saishin Igaku 19: 2459, 1964 (in Japanese).

12) Liebow AA, Carrington CB. The interstitial pneumonias. in: Frontiers of Pulmonary Radiology: Pathophysiologic, Roentogenographic and Radioisotopic Considerations. Simon M, Potchen EJ, May ML, Eds. Grune Stratton, New York, 1969.

13) Colby TV, Carrington CB. Pulmonary lymphoma: Current concepts. Hum Pathol 14: 884, 1983.

14) Sugiyama $Y$, Izumi $T$, Kitamura S, Takaku F. A case of lymphoid interstitial pneumonia accompanied with skin eruption, generalized lymphadenopathy, polyclonal hypergamma-globulinemia and hepatosplenomegaly. Japanese Journal of Thoracic Disease 21: 1083, 1983.

15) Brandt SJ, Bodine DM, Dunbar CE, Nienhuis AW. Dysregulated interleukin 6 expression produces a syndrome resembling Castleman's disease in mice. J Clin Invest 86: 592, 1990.

16) Ohkubo $K$, Ideguchi $H$, Katsuno $M$, et al. A case of plasmacytic lymphadenopathy with polyclonal hypergammaglobulinemia: Evidence for T cell dysfunction. Acta Hematol Jpn 50: 868, 1987. 